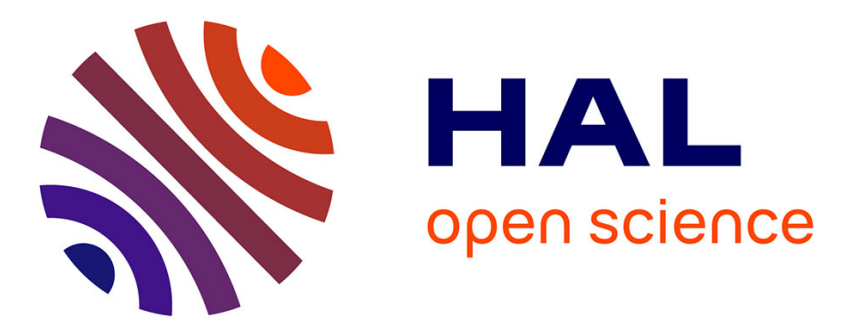

\title{
Adaptation in replicative senescence: a risky business
}

Héloïse Coutelier, Zhou Xu

\section{To cite this version:}

Héloïse Coutelier, Zhou Xu. Adaptation in replicative senescence: a risky business. Current Genetics, 2019, 65 (3), pp.711-716. 10.1007/s00294-019-00933-7 . hal-02325721

\section{HAL Id: hal-02325721 \\ https://hal.science/hal-02325721}

Submitted on 6 Nov 2019

HAL is a multi-disciplinary open access archive for the deposit and dissemination of scientific research documents, whether they are published or not. The documents may come from teaching and research institutions in France or abroad, or from public or private research centers.
L'archive ouverte pluridisciplinaire $\mathbf{H A L}$, est destinée au dépôt et à la diffusion de documents scientifiques de niveau recherche, publiés ou non, émanant des établissements d'enseignement et de recherche français ou étrangers, des laboratoires publics ou privés. 
Title: Adaptation in replicative senescence: a risky business

Authors: Héloïse Coutelier ${ }^{1}$, Zhou $\mathrm{Xu}^{1,2}$

\begin{abstract}
Affiliations:
${ }^{I}$ Sorbonne Université, PSL Research University, CNRS, UMR8226, Institut de Biologie Physico-Chimique, Laboratoire de Biologie Moléculaire et Cellulaire des Eucaryotes, F-75005 Paris, France

${ }^{2}$ Present affiliation: Sorbonne Université, CNRS, UMR7238, Institut de Biologie Paris-Seine, Laboratory of Computational and Quantitative Biology, F-75005 Paris, France.
\end{abstract}

\title{
Correspondence:
}

Email: zhou.xu@sorbonne-universite.fr

Phone number: +331442764 59; (mobile) +3367425 5467

Orcid: 0000-0001-9468-1406

\begin{abstract}
Cell proliferation is tightly regulated to avoid propagating DNA damage and mutations, which can lead to pathologies such as cancer. To ensure genome integrity, cells activate the DNA damage checkpoint in response to genotoxic lesions to block cell cycle progression. This surveillance mechanism provides time to repair the damage before resuming cell cycle with an intact genome. When the damage is not repaired, cells can, in some conditions, override the cell cycle arrest and proceed with proliferation, a phenomenon known as adaptation to DNA damage. A subpopulation of adapted cells might eventually survive, but only at the cost of extensive genome instability. How and in which context adaptation operates the trade-off between survival and genome stability is a fascinating question. After a brief review of the current knowledge on adaptation to DNA damage in budding yeast, we will discuss a new role of adaptation in the context of telomerase-negative cells and replicative senescence. We highlight the idea that, in all settings studied so far, survival through adaptation is a double-edged sword as it comes with increased genomic instability.
\end{abstract}

Keywords: adaptation to DNA damage, telomere, repair, genome instability, Polo kinase.

\section{Acknowledgments}

We thank Maria Teresa Teixeira and Karine Dubrana for their critical reading of the manuscript. This work was supported by "Fondation pour la Recherche Médicale" ("équipe labellisée") and the French National Research Agency (ANR) grant ANR-16-CE12-0026 to Maria Teresa Teixeira in whose lab the work was done, and ANR grant ANR-17-CE20-0002-01 to ZX. HC has been supported by a doctoral grant from the Paris Sciences et Lettres (PSL) Idex program implemented by the ANR (ANR-10-IDEX-0001-02 PSL).

Conflict of interest: The authors declare that they have no conflict of interest. 


\section{DNA damage checkpoint and adaptation}

DNA damage is a major challenge for eukaryotic cells, which must ensure genome integrity while proliferating. The assessment of the damage and the subsequent response are critical for survival and genome stability. Cells have developed a sophisticated safeguard mechanism, called the DNA damage checkpoint, which blocks cellcycle progression to provide time for repair (Weinert and Hartwell 1988; Hartwell and Weinert 1989). Among the variety of DNA lesions, double-strand breaks (DSBs) are particularly cytotoxic if left unrepaired and have served as a model to establish, in budding yeast, the general framework of the DNA damage response, which is conserved throughout eukaryotes. The first step of the DNA damage response is the detection of the break by the independent recruitment of the Ku complex (Yku70/Yu80) and the MRX complex (Mre11/Rad50/Xrs2). They in turn recruit protein kinases of the phosphoinositide 3-kinase-related kinase (PIKK) family, Tell and Mec1, which trigger the DNA damage checkpoint through a cascade of phosphorylation. The culminating step of the cascade is the activation of effector kinases, Rad53 and Chk1, which target multiple cellular processes to ultimately arrest the cell cycle (reviewed in (Finn et al. 2012)).

Two main pathways can repair the damage: non-homologous end joining (NHEJ) and homologous recombination (HR). The decision between these two mechanisms depends on the phase of the cell cycle, through Cdk1 cyclin-dependent kinase activity. NHEJ is favoured in G1 phase and ligates the two broken ends, while HR, which reseals the break using a homologous sequence, is initiated by 5 ' -3 ' resection and exposure of single-stranded DNA (ssDNA) promoted in S/G2 phases.

When repair is successful, the entire DNA damage checkpoint signalling pathway is shut down since the lesion is no longer detected, allowing the cell cycle to resume in a process called recovery. In contrast, when the damage is unrepairable, yeast cells experience a much longer checkpoint arrest ( $\sim 5-15 \mathrm{hrs})$ but can eventually reenter the cell cycle despite the detectable presence of damage (Sandell and Zakian 1993; Toczyski et al. 1997; Lee et al. 1998). This process is known as adaptation to DNA damage. During adaptation, the checkpoint is partially relieved, thus allowing cell cycle progression. More precisely, Rad53 is inactivated, which alleviates the downstream inhibition of cell cycle progression (Pellicioli et al. 2001). The upstream part of the checkpoint remains largely unaffected, including the sensors Ddc1 (subunit of the 9-1-1 clamp complex) and Ddc2 (in complex with Mec1), and the adaptor Rad9, although a very slight drop in Ddc2 foci intensity in a subset of cells and in Rad9 hyperphosphorylation has been reported (Melo et al. 2001; Donnianni et al. 2010; Vidanes et al. 2010).

While many mutants defective for adaptation have been described and have contributed to its characterization, the molecular mechanisms underlying the process are not yet completely elucidated (Harrison and Haber 2006). The conserved family of Polo-like kinases has been implicated in adaptation and recovery to DNA damage checkpoint (Serrano and D'Amours 2014). In budding yeast, Cdc5 is the only Polo kinase and is essential for several cell-cycle-related processes (Botchkarev and Haber 2018). The involvement of Cdc5 in adaptation was first demonstrated with the isolation of the $\mathrm{L} 251 \mathrm{~W}$ point mutant allele, called $c d c 5$-ad, which is defective for adaptation (Toczyski et al. 1997). Cells carrying the $c d c 5-a d$ allele and challenged by an unrepairable DNA damage stay arrested in G2/M and maintain Rad53 in an activated state (Pellicioli et al. 2001). Other recently identified adaptation mutants of CDC5 ( $c d c 5-16$ and $c d c 5-\mathrm{T} 238 \mathrm{~A})$ have also contributed to the dissection of Cdc5's role in adaptation (Ratsima et al. 2011; Ratsima et al. 2016; Rawal et al. 2016). However, despite its central role in adaptation, the exact mechanisms by which $\mathrm{Cdc} 5$ exerts its function and which Cdc5 targets are affected in response to DNA damage are still unclear (Hu et al. 2001; Zhang et al. 2009; Donnianni et al. 2010; Dotiwala et al. 2010; Schleker et al. 2010; Vidanes et al. 2010; Valerio-Santiago et al. 2013; Ratsima et al. 2016; Rawal et al. 2016; Botchkarev et al. 2017).

Not all unrepairable DNA damages seem to be amenable to adaptation. For instance, while a single unrepaired DSB allows adaptation, two simultaneously induced DSBs do not (Lee et al. 1998). One important factor appears to be the amount of single-stranded DNA that accumulates during the arrest, as supported by the fact that the adaptation defect of $y k u 70 \Delta$ mutant, which exhibits increased resection, is suppressed by limiting resection through deletion of MRE11 (Lee et al. 1998). Consistently, cdc13-1 mutant cells placed at restrictive temperature, in which telomeres are uncapped, resected and thus detected as DNA damage, is a widely used setting to study adaptation but the fraction of cells that are able to eventually adapt decreases with increasing temperatures (Toczyski 2006). However, the global amount of ssDNA induced at restrictive temperature in cdc13-1 cells might exceed that found in cells with two DSBs (Garvik et al. 1995; Westmoreland et al. 2018), suggesting that the nature of the damage, and not only the amount of exposed ssDNA, might be important for adaptation. Besides, the $c d c 13-1$-induced telomere dysfunction at higher temperature also involves more complex molecular mechanisms, such as defective nuclear homeostasis of Cdc13-1 proteins and confounding 
temperature-dependent defect of the telomeres themselves (Paschini et al. 2012; Mersaoui and Wellinger 2018), reinforcing the idea that the molecular nature of the damage might impact on adaptation efficiency. Other types of damage, induced by specific treatments, have also been used in vertebrate systems and suggest that adaptation operates only within a range DNA damage load (Yoo et al. 2004; Syljuasen et al. 2006; Rezacova et al. 2011; Kubara et al. 2012).

We recently investigated whether telomerase-negative cells might also undergo adaptation when they activate the DNA damage checkpoint in response to critically short telomeres in a process known as replicative senescence. This new experimental setting we tested is physiologically relevant for human somatic tissues where telomerase is repressed and where cells eventually enter senescence due to telomere exhaustion.

\section{Adaptation in telomere-induced replicative senescence}

Telomeres are repeated sequences found at chromosome extremities. By nature, they resemble one side of a DSB but specific proteins are bound to telomeres and collectively form a capping structure that prevents DNA damage checkpoint activation and unwanted repair by NHEJ or HR (Jain and Cooper 2010; Wellinger and Zakian 2012). The length of a telomere is dynamic and varies with each cell cycle. On the one hand, telomeres shorten due to the DNA-end-replication problem. Conversely, as they become shorter, telomeres can be extended by telomerase, a dedicated reverse transcriptase able to add de novo telomeric repeats. Telomeres are thus kept at a steady-state length distribution, the homeostasis of which is regulated by hundreds of genes (Kupiec 2014; Harari and Kupiec 2018). In the absence of telomerase, telomeres get continuously shorter as cells divide until the shortest one reaches a critical threshold, activating the DNA damage checkpoint and arresting the cells in replicative senescence (Teixeira 2013).

Replicative senescence is a heterogeneous process at several scales. Independent telomerase-negative cultures display variable senescence onset timings. Even within a telomerase-negative cell culture, subcloned single cells give rise to colonies of heterogeneous sizes, reflecting complex cell proliferation dynamics (Lundblad and Szostak 1989; Enomoto et al. 2002). Thus, a telomerase-negative culture is always a mixture of cells at different stages of senescence. The lack of synchrony in reaching senescence precludes the use of standard assays to study the role of adaptation (Toczyski 2006).

To circumvent this issue, we took advantage of a single-cell microfluidics-based method coupled to live microscopy, which previously allowed us to characterize the dynamics of cell divisions in individual cell lineages in which telomerase activity was experimentally repressed (Xu et al. 2015). In particular, we evidenced in individual lineages the presence of frequent cell-cycle arrests that were followed by more cell divisions and were consequently not terminal senescence arrests. We termed them non-terminal arrests and showed that they were, at least partially, Mec1- and Pol32-dependent. Combined with evidence that recombination factors such as Rad51 and Rad52 are important for cell growth not only as an alternative telomere maintenance mechanism but even early after telomerase inactivation (Lundblad and Blackburn 1993; Le et al. 1999; Khadaroo et al. 2009; Churikov et al. 2014; Fallet et al. 2014; Xu et al. 2015; Claussin and Chang 2016; Lue and Yu 2017), this result suggests that telomerase-negative cells frequently experience telomere-related damage, activate the DNA damage checkpoint and undergo repair events such as break-induced replication (BIR).

In a recent report, we demonstrated that, in addition to repair, adaptation is also a relatively frequent response to checkpoint arrests in telomerase-negative cells, occurring in $2-7 \%$ of all cell cycles (Coutelier et al. 2018). Two independent approaches were used: a genetic approach using the adaptation-deficient mutants $c d c 5$-ad and tid1 $1 \Delta$ and a functional approach using a fluorescent reporter to assess cell division with maintenance of the upstream part of the checkpoint pathway, typical of adaptation. Importantly, we did not use strains impaired for repair pathways, as in some adaptation assays (Toczyski 2006). That telomerase-negative cells undergo adaptation even in the presence of functional repair mechanisms begs the question of the cell fate decision between the two pathways. It is possible that the choice simply depends on the type of detected telomeric damage and on how repairable it is. Regarding the nature of the damage, while we do not exclude progressively shortening telomeres reaching a critical threshold as a potential signal, the early timing of the non-terminal compared to terminal arrests makes them hard to reconcile with a model of progressive telomere shortening. We rather speculate that telomere breaks due to replication fork stalling and collapse might trigger these arrests. Whether the molecular nature of the telomere breaks somehow inhibits repair factors and thus channels the cell toward adaptation remains to be investigated further.

Cell survival and genome instability: adaptation as a double-edged sword 
The body of evidence supporting a functional role of adaptation in yeast has grown over the past years. It was initially shown that adaptation is important for survival of irradiated diploid cells when HR is compromised, while at the same time promoting genomic instability in the surviving progeny (Galgoczy and Toczyski 2001). This is supported by a recent study showing that adaptation to genotoxic challenge of HR-deficient diploid cells leads to aneuploid progeny that develops some form of resistance to a second genotoxic challenge (Bender et al. 2018). To follow on a similar idea, in another setting, adaptation is required to survive telomere uncapping by loss of Cdc13 in established telomerase-independent survivors (Mersaoui et al. 2015). The Cdc13-independent cells grow in the presence of permanent damage that is tolerated because of mutations in checkpoint genes. In contrast to surviving telomere uncapping, adaptation is dispensable for the emergence of post senescence telomerase-independent survivors (Coutelier et al. 2018).

While adaptation can be thought of as a last-ditch effort to survive, it is also a strong commitment as the cost of bearing extensive genome instability is great. For instance, the longer cells stay with uncapped telomeres ( $c d c 13-1$ at restrictive temperature), the less viable they are once they return to permissive temperature with functional telomeres, and this loss of viability is partially rescued in an adaptation mutant (Toczyski et al. 1997; Klermund et al. 2014). Maintenance of the checkpoint arrest is thus beneficial for cell survival only if the initial damage is eventually removed or repaired. It is interesting to suggest that adaptation, with its typically long timescale, may be part of a bet edging mechanism to maximize survival in the face of DNA damage with unpredictable persistence. This possibility may be tested experimentally and mathematically modelled.

Our recent study extends the functional role of adaptation to telomerase-negative cells and replicative senescence (Coutelier et al. 2018). After adapting to a prolonged cell-cycle arrest, telomerase-negative cells still had substantial proliferation potential, which added to the relatively high frequency of adaptation events makes postadapted cells a non-negligible fraction of a heterogeneous population of senescing cells. Adaptation is thus a true survival mechanism for telomerase-negative cells experiencing telomere damage. Consistent with the notion that cell proliferation with unrepaired DNA damage is detrimental for genome stability, adaptation contributes to roughly half of the mutation rate observed in senescent cells (Coutelier et al. 2018). Cells undergoing nonterminal arrests followed by adaptation, which occur with increasing frequency as cells divide, would mutate and progressively accumulate in the senescing population. This model partially explains the finding that the mutation rate of telomerase-negative cells increases over time as they lose proliferation potential (Hackett et al. 2001; Hackett and Greider 2003; Coutelier et al. 2018). Because precancerous cells can likewise accumulate high level of genome instability due to dysfunctional telomeres (Maciejowski and de Lange 2017), we suggest that similar adaptation events could occur in aging human cells, contributing to the early stages of tumorigenesis when checkpoints are still functional and leading to genome instability. Consistent with this idea, Plk1 overexpression has been found in numerous human tumours and seems to be involved in neoplastic transformation as well as in resistance to several chemotherapy drugs (Strebhardt and Ullrich 2006; Gutteridge et al. 2016). Plk1-induced genome instability might also be detrimental though if too important, even to cancer cells, as recently reported (de Carcer et al. 2018).

As for other types of damage, adaptation in telomerase-negative cells leads to a wide range of mutations and genome rearrangements (Coutelier et al. 2018). By promoting cell division, adaptation might cause global genome rearrangement starting at the telomeres and initiating a cascade of instabilities through a myriad of possible mechanisms (Galgoczy and Toczyski 2001; Hackett and Greider 2003; Kaye et al. 2004; Vasan et al. 2014; Lopez et al. 2015; Beyer and Weinert 2016; Millet and Makovets 2016). Adapted cells could also explore repair pathways that were not available or utilized during the initial G2/M arrest, such as NHEJ in G1 and BIR or microhomology-mediated end joining (MMEJ) in S/G2 (Galgoczy and Toczyski 2001; Wang et al. 2018). A direct link between the adaptation mechanism and repair pathway choice could be Cdc5/Plk1, since many repair factors are possible substrates for Cdc5 kinase activity, including Sae2 and Mms4 (Donnianni et al. 2010; Matos et al. 2011; Gallo-Fernandez et al. 2012; Schwartz et al. 2012; Szakal and Branzei 2013). Similarly, in human cells, CtIP (ortholog of Sae2), Mre11, Rad51 and BRCA1 are Plk1 targets (Yata et al. 2012; Chabalier-Taste et al. 2016; Li et al. 2017; Wang et al. 2018). Cdc5/Plk1 might therefore preferentially activate some repair pathways while concurrently enabling adaptation.

Overall, adaptation is a double-edged sword for cells experiencing unrepairable DNA damage. The trade-off is that adapted cells may eventually generate a viable progeny but only at the cost of widespread genome instability. The balance between survival and genome instability might depend on the type of damage. Notably, telomere damage in telomerase-negative cells seems amenable to adaptation with substantial survival rate in the progeny, despite extensive genome instability. For unicellular organisms, increased genomic and phenotypic diversity can drive evolution and "adaptation" in a broader sense. However, adapting to DNA damage that could potentially be repaired can be detrimental. In multicellular organisms and in particular mammals, the benefits of 
cell survival through adaptation appear to be largely outweighed by the potential for cancer development, although a positive role of adaptation in the elimination of damaged cells through defective cell cycle progression might also be plausible. The cell fate decision between repair and adaptation is thus critical and although it is achieved, as a first approximation, through timescale separation, more investigation is needed to understand the intricate relationship between adaptation, repair and genome instability. 


\section{References}

Bender K, Vydzhak O, Klermund J, Busch A, Grimm S, Luke B (2018) Checkpoint adaptation in repairdeficient cells drives aneuploidy and resistance to genotoxic agents. bioRxiv doi:10.1101/464685

Beyer T, Weinert T (2016) Ontogeny of Unstable Chromosomes Generated by Telomere Error in Budding Yeast. PLoS Genet 12:e1006345 doi:10.1371/journal.pgen.1006345

Botchkarev VV, Jr., Garabedian MV, Lemos B, Paulissen E, Haber JE (2017) The budding yeast Polo-like kinase localizes to distinct populations at centrosomes during mitosis. Mol Biol Cell 28:1011-1020 doi:10.1091/mbc.E16-05-0324

Botchkarev VV, Jr., Haber JE (2018) Functions and regulation of the Polo-like kinase Cdc5 in the absence and presence of DNA damage. Curr Genet 64:87-96 doi:10.1007/s00294-017-0727-2

Chabalier-Taste C, Brichese L, Racca C, Canitrot Y, Calsou P, Larminat F (2016) Polo-like kinase 1 mediates BRCA1 phosphorylation and recruitment at DNA double-strand breaks. Oncotarget 7:2269-2283 doi:10.18632/oncotarget.6825

Churikov D, Charifi F, Simon MN, Geli V (2014) Rad59-facilitated acquisition of Y' elements by short telomeres delays the onset of senescence. PLoS Genet 10:e1004736 doi:10.1371/journal.pgen.1004736

Claussin C, Chang M (2016) Multiple Rad52-Mediated Homology-Directed Repair Mechanisms Are Required to Prevent Telomere Attrition-Induced Senescence in Saccharomyces cerevisiae. PLoS Genet 12:e1006176 doi:10.1371/journal.pgen.1006176

Coutelier $\mathrm{H}$ et al. (2018) Adaptation to DNA damage checkpoint in senescent telomerase-negative cells promotes genome instability. Genes Dev 32:1499-1513 doi:10.1101/gad.318485.118

de Carcer G et al. (2018) Plk1 overexpression induces chromosomal instability and suppresses tumor development. Nat Commun 9:3012 doi:10.1038/s41467-018-05429-5

Donnianni RA et al. (2010) Elevated levels of the polo kinase Cdc5 override the Mec1/ATR checkpoint in budding yeast by acting at different steps of the signaling pathway. PLoS Genet 6:e1000763 doi:10.1371/journal.pgen.1000763

Dotiwala F, Harrison JC, Jain S, Sugawara N, Haber JE (2010) Mad2 prolongs DNA damage checkpoint arrest caused by a double-strand break via a centromere-dependent mechanism. Current biology : CB 20:328332 doi:10.1016/j.cub.2009.12.033

Enomoto S, Glowczewski L, Berman J (2002) MEC3, MEC1, and DDC2 Are Essential Components of a Telomere Checkpoint Pathway Required for Cell Cycle Arrest during Senescence in Saccharomyces cerevisiae. Mol Biol Cell 13:2626-2638

Fallet E, Jolivet P, Soudet J, Lisby M, Gilson E, Teixeira MT (2014) Length-dependent processing of telomeres in the absence of telomerase. Nucleic Acids Res 42:3648-3665 doi:10.1093/nar/gkt1328

Finn K, Lowndes NF, Grenon M (2012) Eukaryotic DNA damage checkpoint activation in response to doublestrand breaks. Cell Mol Life Sci 69:1447-1473 doi:10.1007/s00018-011-0875-3

Galgoczy DJ, Toczyski DP (2001) Checkpoint adaptation precedes spontaneous and damage-induced genomic instability in yeast. Mol Cell Biol 21:1710-1718

Gallo-Fernandez M, Saugar I, Ortiz-Bazan MA, Vazquez MV, Tercero JA (2012) Cell cycle-dependent regulation of the nuclease activity of Mus81-Eme1/Mms4. Nucleic Acids Res 40:8325-8335 doi:10.1093/nar/gks599

Garvik B, Carson M, Hartwell L (1995) Single-stranded DNA arising at telomeres in cdc13 mutants may constitute a specific signal for the RAD9 checkpoint. Mol Cell Biol 15:6128-6138

Gutteridge RE, Ndiaye MA, Liu X, Ahmad N (2016) Plk1 Inhibitors in Cancer Therapy: From Laboratory to Clinics. Mol Cancer Ther 15:1427-1435 doi:10.1158/1535-7163.MCT-15-0897

Hackett JA, Feldser DM, Greider CW (2001) Telomere dysfunction increases mutation rate and genomic instability. Cell 106:275-286.

Hackett JA, Greider CW (2003) End resection initiates genomic instability in the absence of telomerase. Mol Cell Biol 23:8450-8461

Harari Y, Kupiec M (2018) Do long telomeres affect cellular fitness? Curr Genet 64:173-176 doi:10.1007/s00294-017-0746-z

Harrison JC, Haber JE (2006) Surviving the Breakup: The DNA Damage Checkpoint. Annu Rev Genet 40:209235

Hartwell LH, Weinert TA (1989) Checkpoints: controls that ensure the order of cell cycle events. Science 246:629-634

Hu F, Wang Y, Liu D, Li Y, Qin J, Elledge SJ (2001) Regulation of the Bub2/Bfa1 GAP complex by Cdc5 and cell cycle checkpoints. Cell 107:655-665

Jain D, Cooper JP (2010) Telomeric strategies: means to an end. Annu Rev Genet 44:243-269 doi:10.1146/annurev-genet-102108-134841 
Kaye JA, Melo JA, Cheung SK, Vaze MB, Haber JE, Toczyski DP (2004) DNA breaks promote genomic instability by impeding proper chromosome segregation. Curr Biol 14:2096-2106

Khadaroo B et al. (2009) The DNA damage response at eroded telomeres and tethering to the nuclear pore complex. Nat Cell Biol 11:980-987 doi:10.1038/ncb1910

Klermund J, Bender K, Luke B (2014) High nutrient levels and TORC1 activity reduce cell viability following prolonged telomere dysfunction and cell cycle arrest. Cell Rep 9:324-335 doi:10.1016/j.celrep.2014.08.053

Kubara PM, Kerneis-Golsteyn S, Studeny A, Lanser BB, Meijer L, Golsteyn RM (2012) Human cells enter mitosis with damaged DNA after treatment with pharmacological concentrations of genotoxic agents. Biochem J 446:373-381 doi:10.1042/BJ20120385

Kupiec M (2014) Biology of telomeres: lessons from budding yeast. FEMS Microbiol Rev 38:144-171 doi:10.1111/1574-6976.12054

Le S, Moore JK, Haber JE, Greider CW (1999) RAD50 and RAD51 define two pathways that collaborate to maintain telomeres in the absence of telomerase. Genetics 152:143-152

Lee SE, Moore JK, Holmes A, Umezu K, Kolodner RD, Haber JE (1998) Saccharomyces Ku70, mre11/rad50 and RPA proteins regulate adaptation to G2/M arrest after DNA damage. Cell 94:399-409.

Li Z, Li J, Kong Y, Yan S, Ahmad N, Liu X (2017) Plk1 Phosphorylation of Mre11 Antagonizes the DNA Damage Response. Cancer Res 77:3169-3180 doi:10.1158/0008-5472.CAN-16-2787

Lopez V, Barinova N, Onishi M, Pobiega S, Pringle JR, Dubrana K, Marcand S (2015) Cytokinesis breaks dicentric chromosomes preferentially at pericentromeric regions and telomere fusions. Genes Dev 29:322-336 doi:10.1101/gad.254664.114

Lue NF, Yu EY (2017) Telomere recombination pathways: tales of several unhappy marriages. Curr Genet 63:401-409 doi:10.1007/s00294-016-0653-8

Lundblad V, Blackburn EH (1993) An alternative pathway for yeast telomere maintenance rescues est1senescence. Cell 73:347-360

Lundblad V, Szostak JW (1989) A mutant with a defect in telomere elongation leads to senescence in yeast. Cell 57:633-643 doi:10.1016/0092-8674(89)90132-3

Maciejowski J, de Lange T (2017) Telomeres in cancer: tumour suppression and genome instability. Nat Rev Mol Cell Biol 18:175-186 doi:10.1038/nrm.2016.171

Matos J, Blanco MG, Maslen S, Skehel JM, West SC (2011) Regulatory control of the resolution of DNA recombination intermediates during meiosis and mitosis. Cell 147:158-172 doi:10.1016/j.cell.2011.08.032

Melo JA, Cohen J, Toczyski DP (2001) Two checkpoint complexes are independently recruited to sites of DNA damage in vivo. Genes Dev 15:2809-2821

Mersaoui SY, Gravel S, Karpov V, Wellinger RJ (2015) DNA damage checkpoint adaptation genes are required for division of cells harbouring eroded telomeres. Microbial cell 2:394-405 doi:10.15698/mic2015.10.229

Mersaoui SY, Wellinger RJ (2018) Fine tuning the level of the Cdc13 telomere-capping protein for maximal chromosome stability performance. Curr Genet doi:10.1007/s00294-018-0871-3

Millet C, Makovets S (2016) Aneuploidy as a mechanism of adaptation to telomerase insufficiency. Curr Genet 62:557-564 doi:10.1007/s00294-015-0559-x

Paschini M, Toro TB, Lubin JW, Braunstein-Ballew B, Morris DK, Lundblad V (2012) A naturally thermolabile activity compromises genetic analysis of telomere function in Saccharomyces cerevisiae. Genetics 191:79-93 doi:10.1534/genetics.111.137869

Pellicioli A, Lee SE, Lucca C, Foiani M, Haber JE (2001) Regulation of Saccharomyces Rad53 checkpoint kinase during adaptation from DNA damage-induced G2/M arrest. Mol Cell 7:293-300.

Ratsima H, Ladouceur AM, Pascariu M, Sauve V, Salloum Z, Maddox PS, D'Amours D (2011) Independent modulation of the kinase and polo-box activities of Cdc5 protein unravels unique roles in the maintenance of genome stability. Proc Natl Acad Sci U S A 108:E914-923 doi:10.1073/pnas.1106448108

Ratsima H, Serrano D, Pascariu M, D'Amours D (2016) Centrosome-Dependent Bypass of the DNA Damage Checkpoint by the Polo Kinase Cdc5. Cell Rep 14:1422-1434 doi:10.1016/j.celrep.2016.01.014

Rawal CC, Riccardo S, Pesenti C, Ferrari M, Marini F, Pellicioli A (2016) Reduced kinase activity of polo kinase Cdc5 affects chromosome stability and DNA damage response in S. cerevisiae. Cell Cycle 15:2906-2919 doi:10.1080/15384101.2016.1222338

Rezacova M et al. (2011) Accumulation of DNA damage and cell death after fractionated irradiation. Radiat Res 175:708-718 doi:10.1667/RR2478.1

Sandell LL, Zakian VA (1993) Loss of a yeast telomere: arrest, recovery, and chromosome loss. Cell 75:729-739 
Schleker T, Shimada K, Sack R, Pike BL, Gasser SM (2010) Cell cycle-dependent phosphorylation of Rad53 kinase by $\operatorname{Cdc5}$ and Cdc28 modulates checkpoint adaptation. Cell Cycle 9:350-363 doi:10.4161/cc.9.2.10448

Schwartz EK, Wright WD, Ehmsen KT, Evans JE, Stahlberg H, Heyer WD (2012) Mus81-Mms4 functions as a single heterodimer to cleave nicked intermediates in recombinational DNA repair. Mol Cell Biol 32:3065-3080 doi:10.1128/MCB.00547-12

Serrano D, D'Amours D (2014) When genome integrity and cell cycle decisions collide: roles of polo kinases in cellular adaptation to DNA damage. Systems and synthetic biology 8:195-203 doi:10.1007/s11693-0149151-9

Strebhardt K, Ullrich A (2006) Targeting polo-like kinase 1 for cancer therapy. Nat Rev Cancer 6:321-330 doi:10.1038/nrc1841

Syljuasen RG, Jensen S, Bartek J, Lukas J (2006) Adaptation to the ionizing radiation-induced G2 checkpoint occurs in human cells and depends on checkpoint kinase 1 and Polo-like kinase 1 kinases. Cancer Res 66:10253-10257 doi:10.1158/0008-5472.CAN-06-2144

Szakal B, Branzei D (2013) Premature Cdk1/Cdc5/Mus81 pathway activation induces aberrant replication and deleterious crossover. EMBO J 32:1155-1167 doi:10.1038/emboj.2013.67

Teixeira MT (2013) Saccharomyces cerevisiae as a Model to Study Replicative Senescence Triggered by Telomere Shortening. Front Oncol 3:101 doi:10.3389/fonc.2013.00101

Toczyski DP (2006) Methods for studying adaptation to the DNA damage checkpoint in yeast. Methods Enzymol 409:150-165 doi:10.1016/S0076-6879(05)09009-9

Toczyski DP, Galgoczy DJ, Hartwell LH (1997) CDC5 and CKII control adaptation to the yeast DNA damage checkpoint. Cell 90:1097-1106

Valerio-Santiago M, de Los Santos-Velazquez AI, Monje-Casas F (2013) Inhibition of the mitotic exit network in response to damaged telomeres. PLoS Genet 9:e1003859 doi:10.1371/journal.pgen.1003859

Vasan S, Deem A, Ramakrishnan S, Argueso JL, Malkova A (2014) Cascades of genetic instability resulting from compromised break-induced replication. PLoS Genet 10:e1004119 doi:10.1371/journal.pgen.1004119

Vidanes GM, Sweeney FD, Galicia S, Cheung S, Doyle JP, Durocher D, Toczyski DP (2010) CDC5 inhibits the hyperphosphorylation of the checkpoint kinase Rad53, leading to checkpoint adaptation. PLoS Biol 8:e1000286 doi:10.1371/journal.pbio.1000286

Wang $\mathrm{H}$ et al. (2018) PLK1 targets CtIP to promote microhomology-mediated end joining. Nucleic Acids Res 46:10724-10739 doi:10.1093/nar/gky810

Weinert TA, Hartwell LH (1988) The RAD9 gene controls the cell cycle response to DNA damage in Saccharomyces cerevisiae. Science 241:317-322

Wellinger RJ, Zakian VA (2012) Everything you ever wanted to know about Saccharomyces cerevisiae telomeres: beginning to end. Genetics 191:1073-1105 doi:10.1534/genetics.111.137851

Westmoreland JW, Mihalevic MJ, Bernstein KA, Resnick MA (2018) The global role for Cdc13 and Yku70 in preventing telomere resection across the genome. DNA Repair (Amst) 62:8-17 doi:10.1016/j.dnarep.2017.11.010

Xu Z, Fallet E, Paoletti C, Fehrmann S, Charvin G, Teixeira MT (2015) Two routes to senescence revealed by real-time analysis of telomerase-negative single lineages. Nat Commun 6:7680 doi:10.1038/ncomms 8680

Yata K, Lloyd J, Maslen S, Bleuyard JY, Skehel M, Smerdon SJ, Esashi F (2012) Plk1 and CK2 act in concert to regulate Rad51 during DNA double strand break repair. Mol Cell 45:371-383 doi:10.1016/j.molcel.2011.12.028

Yoo HY, Kumagai A, Shevchenko A, Shevchenko A, Dunphy WG (2004) Adaptation of a DNA replication checkpoint response depends upon inactivation of Claspin by the Polo-like kinase. Cell 117:575-588

Zhang T, Nirantar S, Lim HH, Sinha I, Surana U (2009) DNA damage checkpoint maintains CDH1 in an active state to inhibit anaphase progression. Dev Cell 17:541-551 doi:10.1016/j.devcel.2009.09.006 